\title{
Renal sympathetic denervation alleviates myocardial fibrosis following isoproterenol-induced heart failure
}

\author{
NENG WANG $^{1,2}$, XIAOXIN ZHENG $^{1,2}$, JIN QIAN $^{3}$, WEI YAO $^{3}$, LU BAI $^{3}$, GUO HOU $^{1,2}$, \\ XUAN QIU ${ }^{1,2}$, XIAOYAN LI ${ }^{1}$ and XUEJUN JIANG ${ }^{1,2}$ \\ ${ }^{1}$ Department of Cardiology, Renmin Hospital of Wuhan University; ${ }^{2}$ Cardiovascular Research Institute \\ of Wuhan University, Wuhan, Hubei 430060; ${ }^{3}$ Department of Cardiology, Suizhou Hospital, \\ Hubei University of Medicine, Suizhou, Hubei 441300, P.R. China
}

Received January 17, 2016; Accepted January 20, 2017

DOI: $10.3892 / \mathrm{mmr} .2017 .7255$

\begin{abstract}
The aim of the present study was to determine if renal sympathetic denervation (RSD) may alleviate isoproterenol-induced left ventricle remodeling, and to identify the underlying mechanism. A total of 70 rats were randomly divided into control $(n=15)$, sham operation $(n=15)$, heart failure (HF) with sham operation (HF + sham; $n=20)$ and HF with treatment $(\mathrm{HF}+\mathrm{RSD} ; \mathrm{n}=20)$ groups. The HF model was established by subcutaneous injection of isoproterenol; six weeks later, left ventricular internal diameter at end-systole (LVIDs), left ventricular systolic posterior wall thickness (LVPWs), left ventricular ejection fraction (LVEF) and left ventricular fractional shortening (LVFS) were measured. Plasma norepinephrine (NE), angiotensin II (Ang II) and aldosterone (ALD) levels were measured by ELISA. Myocardial collagen volume fraction (CVF) was determined by Masson's staining. Reverse transcription-quantitative polymerase chain reaction was used to determine the mRNA expression levels of ventricular transforming growth factor- $\beta$ (TGF- $\beta$ ), connective tissue growth factor (CTGF) and microRNAs (miRs), including miR-29b, miR-30c and miR-133a. The results demonstrated that LVIDs and LVPWs in the HF + RSD group were significantly decreased compared with the HF + sham group. By contrast, LVFS and LVEF in the HF + RSD group were significantly increased compared with the HF + sham group. RSD significantly reduced the levels of plasma NE, Ang II and ALD. CVF in the HF + RSD group was reduced by $38.1 \%$ compared with the HF + sham group. Expression levels of TGF- $\beta$ and CTGF were decreased, whereas those
\end{abstract}

Correspondence to: Dr Xuejun Jiang, Department of Cardiology, Renmin Hospital of Wuhan University, 99 Zhang Zhi Dong Street, Wuhan, Hubei 430060, P.R. China

E-mail: xjjiang@whu.edu.cn

Key words: angiotensin II, connective tissue growth factor, microRNA-29b, microRNA-30c, microRNA-133a, renal sympathetic denervation, myocardial fibrosis, norepinephrine, transforming growth factor- $\beta$ of miR-29b, miR-30c and miR-133a were increased, in the $\mathrm{HF}+\mathrm{RSD}$ group compared with the HF + sham group. These results indicated that RSD alleviates isoproterenol-induced left ventricle remodeling potentially via downregulation of TGF- $3 /$ CTGF and upregulation of miR-29b, miR-30c and miR-133a. RSD may therefore be an effective non-drug therapy for the treatment of heart failure.

\section{Introduction}

Chronic congestive heart failure (CHF) is the final outcome of a variety of organic heart diseases. Myocardial fibrosis is the primary form of myocardial remodeling in CHF. It involves the elevation of myocardial interstitial collagen content and alteration of the characteristic collagen configuration, thereby causing increased myocardial rigidity, ventricular systolic and diastolic dysfunction, and decreased coronary flow reserve. Overactivation of the sympathetic nervous system (SNS) and the renin-angiotensin-aldosterone system (RAAS) are important factors responsible for myocardial fibrosis.

Renal sympathetic denervation (RSD) has been used to treat resistant hypertension and associated cardiovascular diseases, including abnormal glucose tolerance and ventricular hypertrophy, caused by chronic SNS and RAAS overactivation. RSD involves the removal of the peripheral portion of the renal artery afferent and efferent nerves, effectively reducing the sympathetic activity under pathological conditions through feedback mechanisms in the hypothalamus central nervous system. RSD has the potential for broad application for the treatment of relevant diseases (1). Systemic sympathetic activation accompanied with increased release of norepinephrine (NE) and angiotensin II (Ang II) promotes transforming growth factor- $\beta$ (TGF- $\beta$ )-mediated myocardial fibrosis. TGF- $\beta 1$ is an important regulatory factor that enhances the expression of connective tissue growth factor (CTGF), which further promotes cell proliferation, extracellular matrix deposition and induction of myocardial fibrosis (2).

MicroRNAs (miRNAs) are endogenous single-stranded non-coding RNAs composed of 21-26 nucleotides. As an important mechanism of epigenetic regulation, miRNAs inhibit the translation of target genes or directly mediate the degradation of target mRNA by binding to the 3 ' untranslated 
region. During heart failure (HF), activation of SNS directly or indirectly (via excited $\beta$-receptor and activated RAAS) regulates miRNA expression, thereby promoting myocardial remodeling. Inhibition of the sympathetic activity may suppress myocardial remodeling by regulating the expression levels of miRNA. Previous studies have demonstrated that miRNA (miR)-29, miR-30 and miR-133 are the primary regulators of TGF- $\beta$ and CTGF, and are closely associated with the occurrence of myocardial fibrosis (3).

A primary strategy for the treatment of HF is the suppression of excessive SNS and RAAS activation and the inhibition of myocardial fibrosis. Renal sympathetic activation is a common mechanism underlying pathophysiology in HF and high blood pressure. A pilot trial has reported that RSD significantly improves cardiac function in HF patients with no adverse effects on blood flow and renal function, suggesting that RSD is a potential non-drug approach for the treatment of HF (4). The present study established the isoproterenol-induced HF model in rats (5) and determined the effect of RSD on myocardial fibrosis. In addition, the underlying molecular mechanisms by which RSD inhibits myocardial fibrosis were investigated, with the aim of providing a theoretical basis for the use of RSD as a potential treatment for HF.

\section{Materials and methods}

Experimental animals. Sprague-Dawley male rats (weight, 250-350 g; $\mathrm{n}=70$ ) were purchased from Beijing HFK Bioscience Co, Ltd. (Beijing, China). Rats were housed under a 12-h light/dark cycle at a temperature of $23 \pm 2^{\circ} \mathrm{C}$ and a humidity of $61 \pm 5 \%$. Animals were allowed to acclimatize for at least one week prior to the experiment. All animal procedures complied with IACUC (Institutional Animal Care and Use Committee) of Wuhan University (Wuhan, China).

Animal groups. Rats were randomly divided into four groups: Control $(n=15)$, in which the rats were untreated; sham $(n=15)$, in which a sham procedure was performed on the rats; HF + sham $(n=20)$, in which a sham procedure was performed on the rats followed by HF induction; and HF + RSD ( $n=20)$, in which RSD was performed on the rats followed by HF induction.

RSD and HF-induction. An intraperitoneal injection of $40 \mathrm{mg} / \mathrm{kg}$ sodium pentobarbital (Sigma-Aldrich; Merck KGaA, Darmstadt, Germany) was performed to anesthetize the rats at room temperature. Subsequently, abdominal skin was disinfected, covered with towels and a midline laparotomy was performed. Subcutaneous tissues and muscles were separated layer by layer to expose the kidney, ureter, intrathecal artery, vein and nerve. The sheath of the artery and vein was stripped and all visible nerve fibers were disarticulated under a dissecting microscope (magnification, x25). A cotton ball soaked with a $95 \%$ ethanol and $10 \%$ phenol solution was applied to the renal artery and vein to achieve sufficient denervation. The renal nerve electrical stimulation method was used to evaluate if renal denervation was effective, as described previously (6). Prior to sympathetic denervation, electrical stimulation (15 V, $0.2 \mathrm{msec}, 10 \mathrm{~Hz}$, square wave) was applied at the proximal end of the renal nerve for $10-30 \mathrm{sec}$ to induce a significant sympathetic response (a systolic blood pressure increase of 5-10 mmHg, heart rate increase of 8-15 bpm and pale kidneys). By contrast, following complete denervation, electrical stimulation did not produce these effects. Strict aseptic conditions were used, and 80,000 U/day penicillin was injected intraperitoneally to prevent infection. A slow subcutaneous injection of $4 \mathrm{mg} / \mathrm{kg}$ isopropyl adrenaline (ISO) was performed once a day for 10 consecutive days to induce HF.

Echocardiography. A total of six weeks after the successful establishment of the HF model, Doppler echocardiography was performed on the surviving rats. Rats were anesthetized by an intraperitoneal injection of $2 \%$ sodium pentobarbital $(40 \mathrm{mg} / \mathrm{kg}$ ). The rats were then fixed in a supine position and a parasternal short-axis view was obtained. M-mode echocardiography was used to record the left ventricular systolic and diastolic motion profile. The left ventricular internal diameter (LVIDs), left ventricular systolic posterior wall thickness (LVPWs), left ventricular ejection fraction (LVEF) and left ventricular fractional shortening (LVFS) were measured.

Measurement of plasma norepinephrine, angiotensin II and aldosterone levels. Following the ultrasound examination at the endpoint of the experiment, peripheral blood samples were obtained and coagulation was prevented with 10\% EDTA and aprotinin. Following centrifugation at $1,200 \mathrm{x} \mathrm{g}$ at $4^{\circ} \mathrm{C}$ for $20 \mathrm{~min}$, plasma samples were stored at $-70^{\circ} \mathrm{C}$. NE, Ang II and ALD levels were measured by ELISA, according to the manufacturer's protocol (rat kit; cat. nos. ab47831 and ab136933; Abcam, Cambridge, UK).

Masson's staining. At the experimental endpoint, animals were sacrificed by intraperitoneal injection of an overdose of sodium pentobarbital. Ventricular tissue was harvested, fixed, paraffin-embedded and sections into $5 \mu \mathrm{m}$ were prepared for Masson's staining (7). A Leica-Q500MC image analyzer (Leica Microsystems GmbH, Wetzlar, Germany) was used to determine the myocardial fibrosis area and to calculate the percentage collagen volume fraction (CVF).

Immunohistochemical detection of TGF- $\beta$ and CTGF. Expression of TGF- $\beta$ and CTGF was determined by immunohistochemistry as described previously (8). Briefly, following dehydration of the paraffin sections, primary mouse monoclonal antibodies against TGF- $\beta$ and CTGF (1:50; catalog nos. SAB4502958 and HPA031074; Sigma-Aldrich; Merck KGaA) were added at $4^{\circ} \mathrm{C}$ overnight. Following a thorough wash, a horseradish peroxidase (HRP)-conjugated secondary antibody (1:400; cat. no. A0545; goat anti-rabbit IgG; Sigma-Aldrich; Merck KGaA) was applied. Image-Pro Plus software version 6.0 (Media Cybernetics, Inc., Rockville, MD, USA) was used determine the average optical density.

Reverse transcription-quantitative polymerase chain reaction (RT-qPCR) analysis. Myocardial cells were collected and total RNA was isolated using RNAiso Plus (Takara Biotechnology Co., Ltd., Dalian, China). cDNA was obtained by RT using the First-Strand cDNA Synthesis kit (Toyobo Co., Ltd., Osaka, Japan) and oligo (dT) $)_{15}$. qPCR was subsequently performed using the FastStart Universal SYBR ${ }^{\circledR}$-Green Master kit (Roche Applied Science, Mannhein, Germany) in a $10 \mu 1$ reaction 
Table I. Primer sequences for reverse transcription-quantitative polymerase chain reaction.

\begin{tabular}{lllr}
\hline Primer & \multicolumn{1}{c}{ Forward } & \multicolumn{1}{c}{ Reverse } & Length \\
\hline rat(RT)-TGF- $\beta$ & GCGCCTGCAGAGATTCAAGTC & GGCGTATCAGTGGGGGTCAG & 113 \\
rat(RT)-CTGF & GGCCCTGTGAAGCTGACCTAG & GCCCGGTAGGTCTTCACACTG & 124 \\
GAPDH & GGCACAGTCAAGGCTGAGAATG & ATGGTGGTGAAGACGCCAGTA & 143 \\
\hline
\end{tabular}

TGF- $\beta$, transforming growth factor- $\beta$; CTGF, connective tissue growth factor; RT, reverse transcription.

volume on an ABI 7900 Real-Time PCR system (Applied Biosystems; Thermo Fisher Scientific, Inc., Waltham, MA, USA). The reaction mixture contained $2 \mu \mathrm{l}$ cDNA template, $5 \mu$ l SYBR-Green (including ROX) mix, $200 \mathrm{nM}$ forward primer and $200 \mathrm{nM}$ reverse primer (9). The mRNA level of TGF- $\beta$ and CTGF were measured. Human GAPDH served as an internal standard. The primers for RT-PCR analyses are listed in Table I. The expression levels of miR-29b, miR-30c and miR-133a were measured using corresponding Bulge-Loop miRNA qRT-PCR Starter kits (Guangzhou RibiBio Co., Ltd., Guangzhou, China). miR-29b, miR-30c and miR-133a were extracted from total RNA and their expression levels were measured. U6 snRNA qRT-PCR Primer Set (Guangzhou Ribobio Co., Ltd) served as an internal reference. The PCR products were verified by melt curve analysis and the results were analyzed using the $2^{-\Delta \Delta \mathrm{Cq}}$ method $\left(\mathrm{RQ}=2^{-\Delta \Delta \mathrm{Cq}}, \Delta \Delta \mathrm{C}_{\mathrm{q}}\right.$ is for the individual, $\Delta \mathrm{C}_{\mathrm{q}}$ is the calibrator) (10). Each assay was performed in triplicate and repeated at least three times.

Western blot analysis. Myocardial tissue samples were collected and incubated in ice-cold TNEN lysis buffer $(50 \mathrm{mM}$ Tris- $\mathrm{HCl}, \mathrm{pH}$ 7.5, $150 \mathrm{mM} \mathrm{NaCl}, 2.0 \mathrm{mM}$ EDTA and $1.0 \%$ Nonidet P-40) with a mini tablet of EDTA-free protease inhibitors (Roche Applied Science) and $1 \mathrm{mmol} / 1$ phenylmethylsulfonyl fluoride for $30 \mathrm{~min}$ at $4^{\circ} \mathrm{C}$. The insoluble fraction was pelleted by centrifugation at $12,000 \mathrm{x}$ g for $15 \mathrm{~min}$ at $4^{\circ} \mathrm{C}$. $100 \mu \mathrm{l}$ supernatant was mixed with $20 \mu \mathrm{l} 6 \mathrm{X}$ laemmli buffer (0.3 mol/1 Tris-HCl, 6\% SDS, 60\% glycerol, $120 \mathrm{mmol} / 1$ dithiothreitol and proprietary pink tracking dye), and heated at $37^{\circ} \mathrm{C}$ for $10 \mathrm{~min}$. Subsequently, $20 \mu 1$ samples $(1 \mu \mathrm{g} / \mu 1$ protein) were subjected to $10 \%$ SDS-PAGE. Following electrophoresis, proteins were transferred onto a $0.45 \mu \mathrm{m}$ PVDF membrane (EMD Millipore, Billerica, MA, USA). The membrane was probed with anti-TGF- $\beta$ and anti-CTGF rabbit polyclonal antibodies (1:500; cat. nos. SAB4502958 and HPA031074; Sigma-Aldrich; Merck KGaA) at $4^{\circ} \mathrm{C}$ overnight, followed by incubation with a horseradish peroxidase-conjugated secondary goat anti-rabbit antibody (1:5,000; cat. no. A0545; Sigma-Aldrich; KGaA) for $4 \mathrm{~h}$ at room temperature. The protein signal was visualized using the Super Signal West Pico Chemiluminescent substrate (Thermo Fisher Scientific, Inc.), according to the manufacturer's protocol. Human GAPDH (rat; 1:3,000; cat. no. G9545; Sigma-Aldrich; KGaA) served as a loading control. Each assay was performed in triplicate and repeated at least three times.

Statistical analysis. SPSS software version 17.0 (SPSS, Inc., Chicago, IL, USA) was used for statistical analysis. Data are
Table II. Effect of RSD on NE, Ang II and ALD levels in rats with isoproterenol-induced HF.

\begin{tabular}{lcccc}
\hline Group & $\mathrm{n}$ & $\begin{array}{c}\text { Plasma NE } \\
(\mathrm{pg} / \mathrm{ml})\end{array}$ & $\begin{array}{c}\text { Plasma Ang II } \\
(\mathrm{pg} / \mathrm{ml})\end{array}$ & $\begin{array}{c}\text { Plasma ALD } \\
(\mathrm{pg} / \mathrm{ml})\end{array}$ \\
\hline Control & 15 & $159.3 \pm 11.1$ & $208.5 \pm 14.3$ & $205.3 \pm 14.8$ \\
Sham & 13 & $168.2 \pm 13.7$ & $201.6 \pm 10.5$ & $197.0 \pm 9.5$ \\
HF + sham & 12 & $264.6 \pm 13.6$ & $491.1 \pm 14.8^{\mathrm{a}}$ & $517.3 \pm 16.0^{\mathrm{a}}$ \\
HF + RSD & 10 & $200.7 \pm 8.0$ & $388.3 \pm 13.7^{\mathrm{b}}$ & $408.5 \pm 12.3^{\mathrm{b}}$
\end{tabular}

Data are expressed as the mean \pm standard deviation. ${ }^{\mathrm{a}} \mathrm{P}<0.01$ vs. control and sham groups; ${ }^{b} \mathrm{P}<0.01 \mathrm{vs}$. $\mathrm{HF}+$ sham group. RSD, renal sympathetic denervation; NE, norepinephrine; Ang II, angiotensin II; ALD, aldosterone; HF, heart failure.

expressed as the mean \pm standard deviation. One-way analysis of variance was conducted to compare experimental groups, followed by Scheffe's post hoc test. $\mathrm{P}<0.05$ was considered to indicate a statistically significant difference.

\section{Results}

Overall animal survival. A total of 20 rats died during the present study, including 4 unexpected deaths from anesthesia and 16 heart failure-associated deaths following subcutaneous injection of drugs. The overall survival rate of the rats was $62.5 \%$. The remaining animals survived to endpoint with 15 rats in the control, 13 rats in the sham, 12 rats in the $\mathrm{HF}+$ sham and 10 rats in the HF + RSD groups.

Echocardiography. In the HF + sham group, LVIDs and LVPWs were significantly increased, whereas LVFS was significantly decreased compared with the sham group $(\mathrm{P}<0.05)$, suggesting that the HF model was successfully established in the rats. These cardiac parameters were significantly improved in the $\mathrm{HF}+\mathrm{RSD}$ group $(\mathrm{P}<0.05$; Fig. 1$)$, indicating that RSD is effective in improving heart function.

Effect of RSD on plasma NE, Ang II and ALD levels. NE, Ang II and ALD levels in the $\mathrm{HF}+$ sham group were significantly greater compared with the sham group $(\mathrm{P}<0.01$; Table II). By contrast, the concentration of NE, Ang II and ALD in the HF + RSD group was significantly reduced compared with the $\mathrm{HF}+$ sham group $(\mathrm{P}<0.01)$. There was no significant difference between the sham and control groups. 

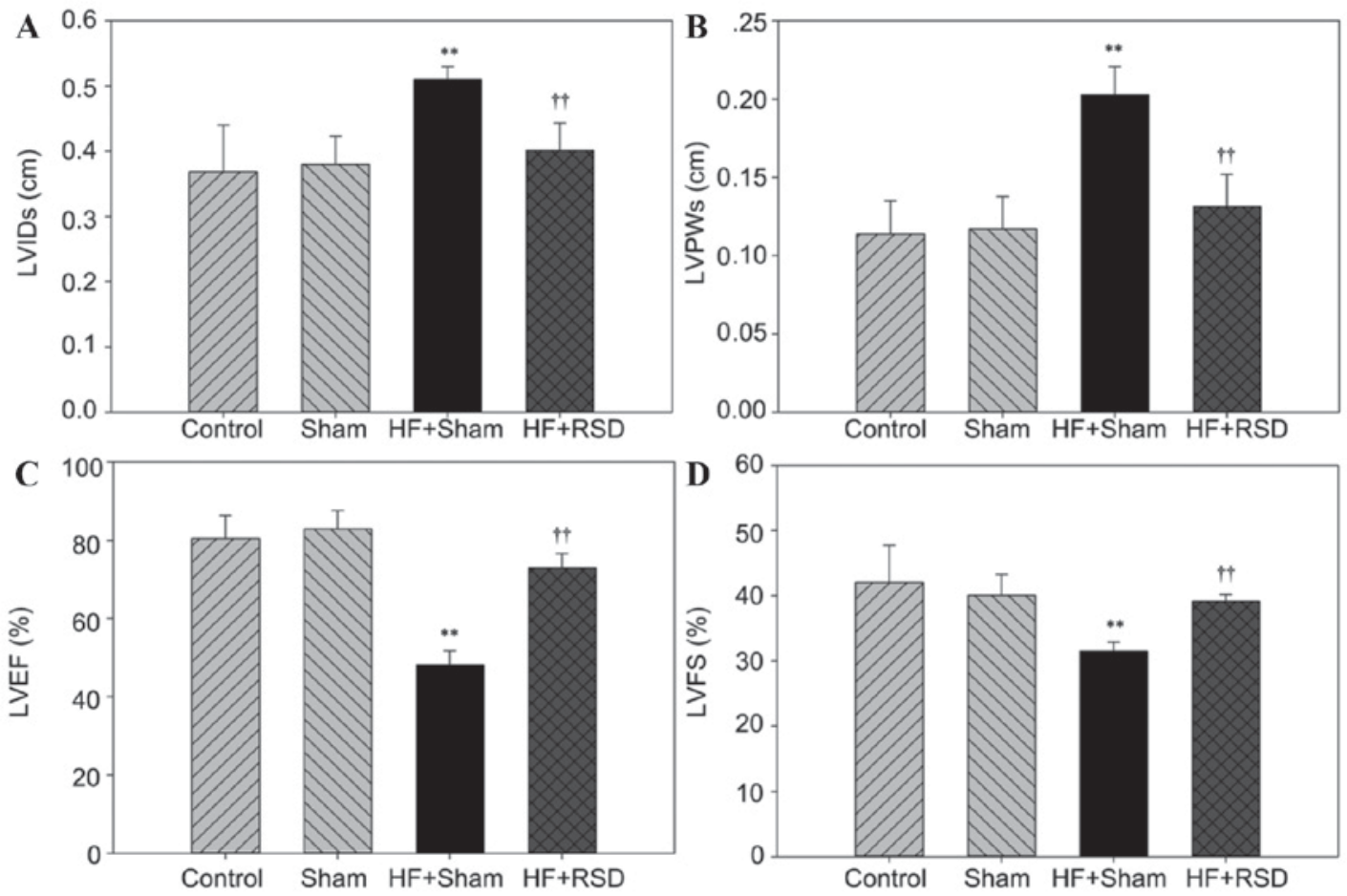

Figure 1. Comparison of cardiac functional parameters among the groups of rats. (A) LVIDs, (B) LVPWs, (C) LVEF and (D) LVFS were measured in control, sham, HF + sham and HF + RSD groups. Data are expressed as the mean \pm standard deviation. ${ }^{* *} \mathrm{P}<0.01$ vs. control and sham groups; ${ }^{\dagger \dagger} \mathrm{P}<0.01 \mathrm{vs}$. $\mathrm{HF}+\mathrm{sham}$ group. LVIDs, left ventricular internal diameter at end-systole; LVPWs, left ventricular systolic posterior wall thickness; LVEF, 1eft ventricular ejection fraction; LVFS, left ventricular fractional shortening; RSD, renal sympathetic denervation; HF, heart failure.
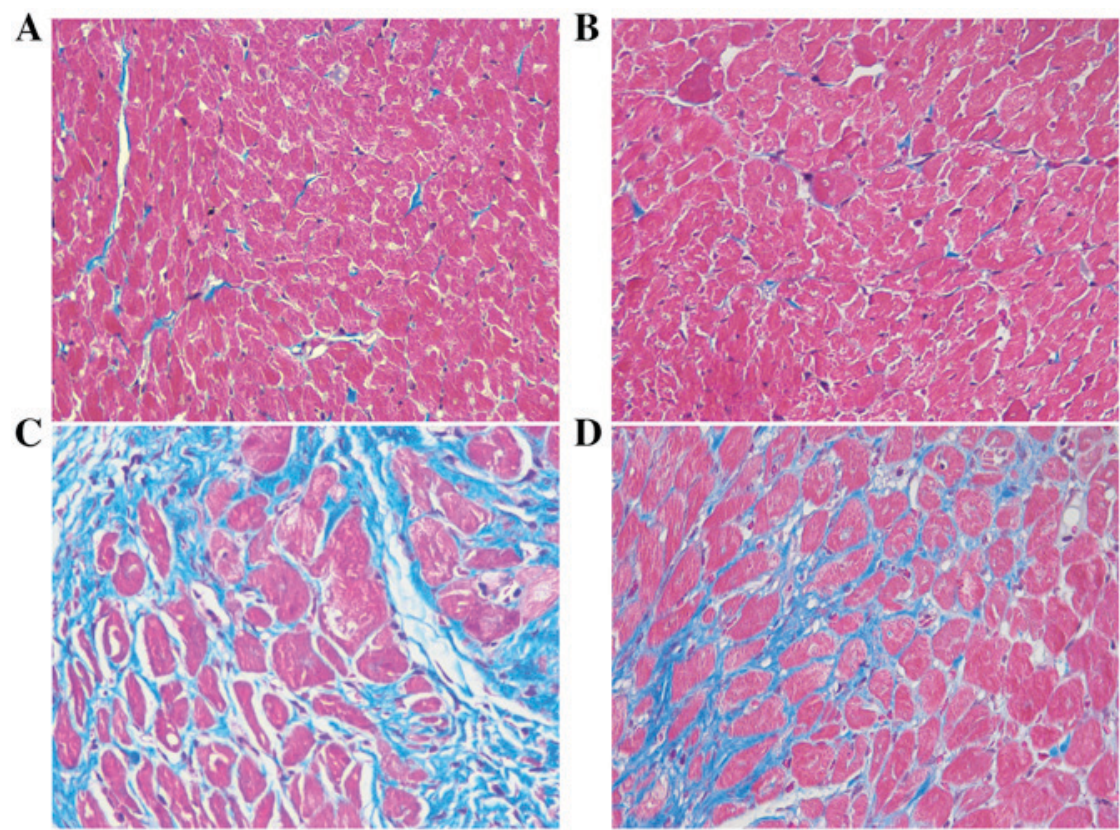

Figure 2. Masson's staining of collagen deposition. (A) Control (B) Sham (C) HF + Sham (D) HF + RSD. CVF values were as follows: Control 4.94 $\pm 0.38 \%$; Sham 4.67 \pm 0.63\%; HF + Sham 60.76 $\pm 6.01 \%$ and HF + RSD 43.99 $\pm 6.23 \%$. Normal myocardial tissues are depicted in red and collagen is depicted in blue. (Original magnification, $\mathrm{x} 400$ ). CVF, collagen volume fraction; RSD, renal sympathetic denervation; HF, heart failure.

These results indicated that RSD may reduce the levels of NE, Ang II and ALD during HF.

Effect of RSD on CVF. As determined by Masson's staining (Fig. 2), CVF in the HF + sham group was increased by 1,230 and $1,300 \%(\mathrm{P}<0.01)$, compared to that in the control and sham groups, respectively. CVF in the $\mathrm{HF}+\mathrm{RSD}$ group was reduced by $38.1 \%(\mathrm{P}<0.01)$ compared with the $\mathrm{HF}+$ sham group (Fig. 2), indicating that RSD may reduce CVF during HF.

Effect of RSD on the expression of TGF- $\beta 1$ and CTGF. RT-qPCR revealed that TGF- $\beta$ (Fig. 3A) and CTGF (Fig. 3B) 

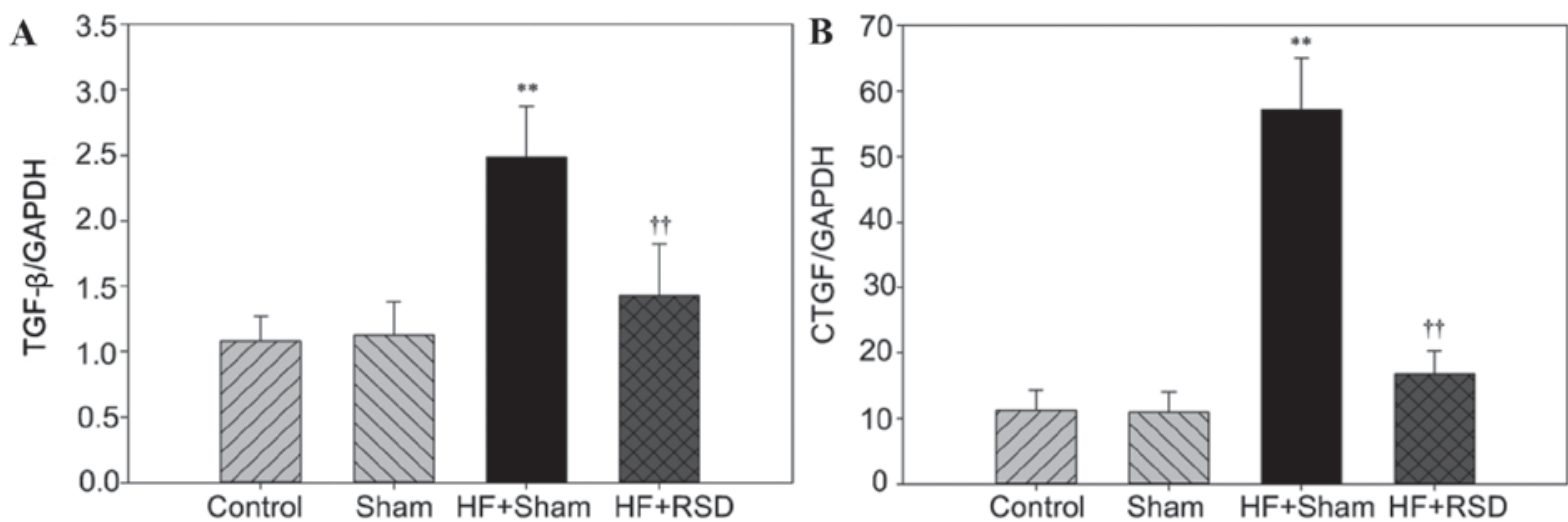

Figure 3. mRNA expression levels of TGF- $\beta$ and CTGF in myocardial tissues 6 weeks after establishing HF, as determined by reverse transcription-quantitative polymerase chain reaction. Compared with the HF + sham group, the HF + RSD group had significantly reduced mRNA expression levels of (A) TGF- $\beta$ and (B) CTGF. Data are expressed as the mean \pm standard deviation. ${ }^{* *} \mathrm{P}<0.01 \mathrm{vs}$. control and sham groups; ${ }^{\dagger \dagger} \mathrm{P}<0.01 \mathrm{vs}$. HF + sham group. TGF- $\beta$, transforming growth factor- $\beta$; CTGF, connective tissue growth factor; HF, heart failure; RSD, renal sympathetic denervation.
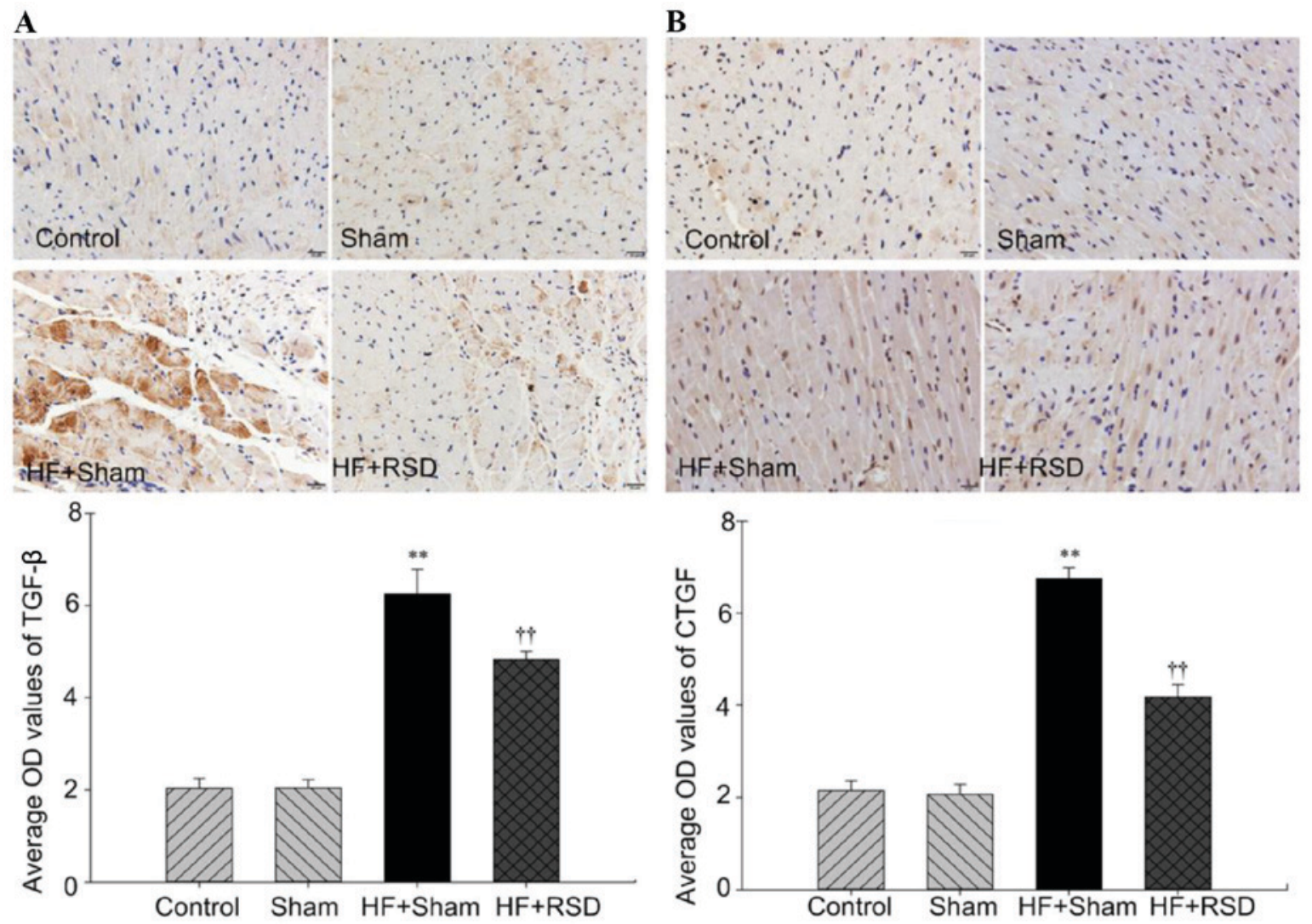

Figure 4. Immunohistochemical analysis of TGF- $\beta$ and CTGF in myocardial tissues. Positive staining of (A) TGF- $\beta$ and (B) CTGF in each group appears brown. Hematoxylin nuclear staining appeared blue. In the HF + RSD group, positive staining of TGF- $\beta$ and CTGF was significantly reduced compared with the $\mathrm{HF}+$ sham group. Data are expressed as the mean \pm standard deviation. ${ }^{* *} \mathrm{P}<0.01$ vs. sham group; ${ }^{\circ} \mathrm{P}<0.01$ vs. $\mathrm{HF}+$ sham group; TGF- $\beta$, transforming growth factor- $\beta$; CTGF, connective tissue growth factor; $\mathrm{HF}$, heart failure; RSD, renal sympathetic denervation.

mRNA expression levels in the HF + sham group were significantly greater compared with the control and sham groups $(\mathrm{P}<0.01)$. mRNA expression levels of TGF- $\beta 1$ and CTGF in the $\mathrm{HF}+\mathrm{RSD}$ group were significantly reduced compared with the $\mathrm{HF}+$ sham group $(\mathrm{P}<0.01)$. Immunohistochemical analysis demonstrated greater staining of TGF-b1 (Fig. 4A) and CTGF (Fig. 4B) in the HF + sham group compared with the control and sham groups $(\mathrm{P}<0.01)$; staining was reduced by RSD treatment $(\mathrm{P}<0.01)$. Additionally, western blot analysis revealed that TGF- $\beta$ (Fig. 5A) and CTGF (Fig. 5B) protein expression levels were significantly reduced following RSD treatment compared with the sham treatment.

Effect of RSD on the expression levels of $m i R-29 b, m i R-30 c$ and miR-133a. The expression levels of miR-29b, miR-30c and miR-133a in the HF + sham group were significantly reduced compared with the sham group [miR-29b, 5.87 \pm 0.97 vs. $30.94 \pm 4.65(\mathrm{P}<0.01)$; miR-30c, $0.28 \pm 0.16$ vs. $1.46 \pm 0.20$ 
A
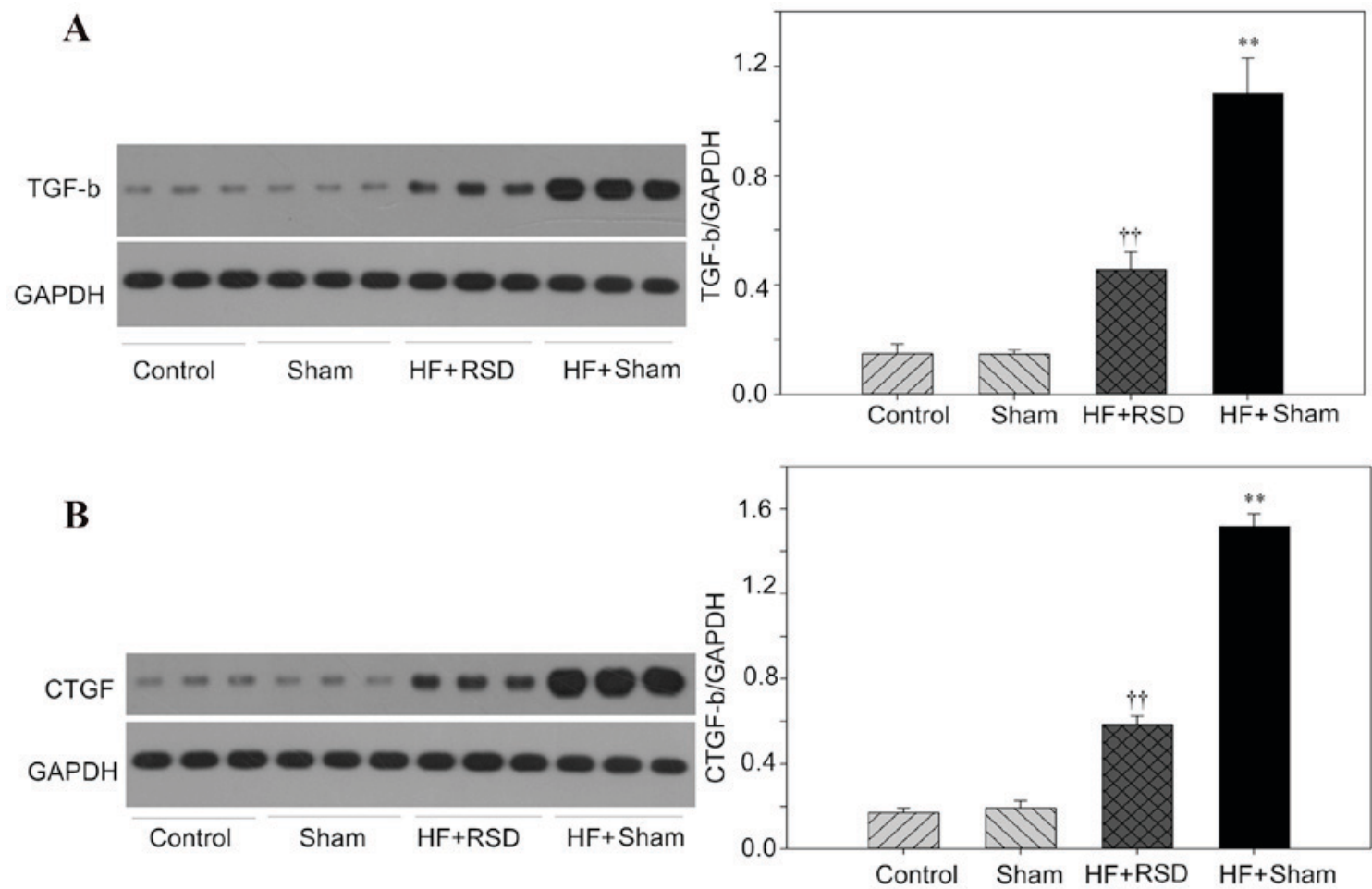

Figure 5. Western blot analysis of TGF- $\beta$ and CTGF protein expression levels in myocardial tissues. Protein expression levels of (A) TGF- $\beta$ and (B) CTGF in myocardial tissues were determined 6 weeks after the establishment of HF. Compared with the HF + sham group, TGF- $\beta$ and CTGF protein expression levels in the HF + RSD group were significantly reduced. Data are expressed as the mean \pm standard deviation. The expression levels of TGF- $\beta$ were as follows: Control, $0.15 \pm 0.03$; sham, $0.15 \pm 0.01 ; \mathrm{HF}+\mathrm{RSD} 0.46 \pm 0.06$ and HF + sham $1.10 \pm 0.02$. The expression levels of CTGF were as follows: Control, $0.17 \pm 0.03$; sham, $0.19 \pm 0.04 ; \mathrm{HF}+\mathrm{RSD} 0.58 \pm 0.04$ and $\mathrm{HF}+$ sham $1.52 \pm 0.06 .{ }^{* *} \mathrm{P}<0.01$ vs. sham group; ${ }^{\dagger} \mathrm{P}<0.01 \mathrm{vs}$. HF + sham group; TGF- $\beta$, transforming growth factor- $\beta$; CTGF, connective tissue growth factor; HF, heart failure; RSD, renal sympathetic denervation.
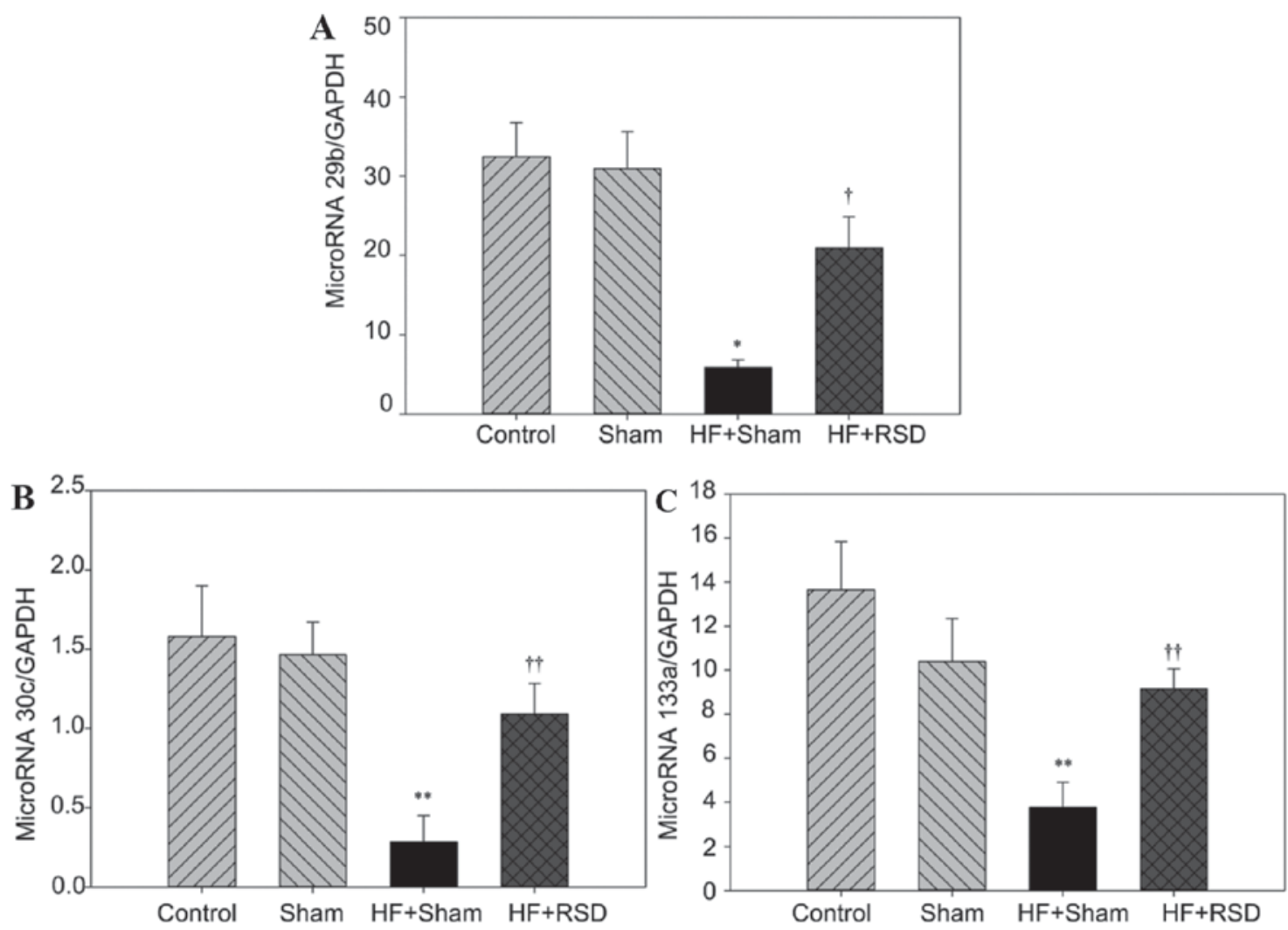

Figure 6. Expression levels of miR-29b, miR-30c and miR-133a in myocardial tissues. Compared with the HF + sham group, expression of (A) miR-29b, (B) miR-30c and (C) miR-133a in the HF + RSD group was significantly greater. Data are expressed as the mean \pm standard deviation. "P<0.05 vs. control and sham groups; ${ }^{* *} \mathrm{P}<0.01$ vs. control and sham groups; ${ }^{\mathrm{P}}<0.05 \mathrm{vs}$. $\mathrm{HF}+$ sham group; ${ }^{\dagger} \mathrm{P}<0.01$ vs. HF + sham group. miR, microRNA; HF, heart failure; RSD, renal sympathetic denervation. 
( $\mathrm{P}<0.01)$; miR-133a, 3.76 \pm 1.14 vs. $10.39 \pm 1.95$ (P<0.01; Fig. 6)]. Expression levels of miR-29b, miR-30c and miR-133a in the $\mathrm{HF}+\mathrm{RSD}$ group were significantly greater compared with the $\mathrm{HF}+$ sham group [miR-29b, 21.69 \pm 3.12 vs. 5.87 $\pm 0.97(\mathrm{P}<0.01)$; miR-30c, $1.09 \pm 0.19$ vs. $0.28 \pm 0.16(\mathrm{P}<0.01)$; miR-133a, $9.14 \pm 0.90$ vs. $3.76 \pm 1.14$ ( $\mathrm{P}<0.01$; Fig. 6)]. miRNA expression levels were not significantly different between the control and sham groups $(\mathrm{P}>0.05)$.

\section{Discussion}

The present study demonstrated that RSD may effectively reduce LVIDs and LVPWs, and increase LVEF and LVFS, leading to the improvement of left ventricular function in the ISO-induced rat HF model. These results are consistent with a previous report, which suggested that angiotensin-converting enzyme inhibitors may alleviate ventricular remodeling via a reduction of ventricular volume overload (11).

An increase in synthesis or decrease in degradation of myocardial collagen serves an important role in myocardial rigidity following $\mathrm{HF}$, which ultimately affects left ventricular function. The present study demonstrated that myocardial CVF in the HF group was significantly increased compared with the control and sham groups, indicating that ventricular fibroblasts proliferate, leading to increased synthesis and secretion of collagen as well as remodeling of the cardiac collagen spatial network. Reduction of CVF in the RSD treatment group indicated that ventricular collagen deposition decreased, thereby increasing ventricular adaptability. The underlying mechanisms by which RSD reduces CVF require further investigation.

TGF- $\beta$ and CTGF are the two most important factors in promoting myocardial fibrosis. TGF- $\beta$ is a multi-functional polypeptide involved in cell proliferation, differentiation, migration, apoptosis and matrix formation. In chronic infection, TGF- $\beta$ is activated, which promotes the formation of muscle fibroblasts. This inhibits extracellular matrix degradation and increases extracellular matrix mRNA expression and protein synthesis. Thus, TGF- $\beta$ serves an important role in the development of myocardial fibrosis (12). CTGF may promote fibroblast cell division and collagen deposition (13). CTGF is an important marker of myocardial fibrosis and is regarded as a downstream mediator of TGF- $\beta$, as it has numerous biological similarities to TGF- $\beta$. CTGF may promote cell proliferation and collagen synthesis, induce extracellular matrix deposition and inhibit its degradation. In addition, CTGF may promote the expression of protease inhibitors, including plasminogen activator inhibitor and tissue inhibitor of metalloproteinase (14). Furthermore, CTGF may promote angiogenesis and facilitate myocardial fibrosis (15) via cell adhesion and aggregation (16). The results of the present study demonstrated that RSD significantly reduced the expression of TGF- $\beta$ and CTGF, which may contribute to the improvement of cardiac function.

miRNAs have been demonstrated to serve an important role in epigenetic regulation. To date, 1,424 miRNAs have been identified in humans, which regulate $>60 \%$ of proteins. miRNAs serve a role in myocardial remodeling in HF. For example, miR-29, miR-133 and miR-30 have been demonstrated to be involved in myocardial fibrosis (17). The present study further investigated the effects of RSD on the expression of miRNA in myocardial tissues. RSD increased the expression of miR-29b, miR-30c and miR-133a in rats with HF. It has previously been demonstrated that SNS activation may increase TGF- $\beta$-mediated myocardial fibrosis via the release of NE (18). In addition, SNS activation may upregulate Ang II levels and stimulate the synthesis of TGF- $\beta$ (19). Ang II enhances the synthesis of CTGF via upregulation of RacI, which further promotes myocardial fibrosis (20). Perlini et al (21) revealed that sympathetic denervation may inhibit myocardial fibrosis. miR-29 is primarily present in myocardial fibroblasts. Numerous extracellular matrix-associated genes, including elastin, fibrillin-1, collagen (COL) type I $\alpha 1$ chain, COL type I $\alpha 2$ chain and COL type III $\alpha 1$ chain are regulated by miR-29. A reduction in miR-29 expression may promote myocardial interstitial fibrosis (22); TGF- $\beta$ may downregulate the expression of miR-29, leading to myocardial fibrosis. As an important factor in promoting myocardial fibrosis, CTGF is regulated by miR-133 and miR-30. Overexpression of miR-133 and miR-30 may reduce the expression of CTGF, thereby inhibiting myocardial fibrosis (23). Therefore, growth factors and associated miRNAs produce a complete feedback loop in the regulation of ventricular remodeling, which may be partially responsible for the underlying mechanism by which RSD inhibits ventricular remodeling in HF.

SNS activation is closely associated with the prognosis of HF. Following SNS activation, the release of NE and neuropeptide $\mathrm{Y}$ is increased, causing peripheral vasoconstriction. This reduces renal blood flow and excretion of sodium and water, and promotes the process of HF (24). In addition to the direct effect on hemodynamics, SNS activation is closely associated with myocardial fibrosis. Wang et al (25) suggested that RSD may significantly reduce the concentration of Ang II and ALD and inhibit atria remodeling in a canine model of HF induced by rapid pacing. Clayton et al (26) demonstrated that RSD may inhibit myocardial expression of the Ang II receptor, increase urinary sodium excretion, reduce plasma brain natriuretic peptide levels and improve cardiac functions in a rabbit HF model. A study performed by $\mathrm{Hu}$ et al (27) indicated that RSD may significantly improve cardiac remodeling, increase urine volume and improve cardiac function in a rat model of myocardial infarction. Therefore, reduction of neurohormone activation status by the simultaneous inhibition of SNS and RAAS may be a primary underlying mechanism by which RSD improves cardiac function. This hypothesis may explain the effect of RSD on growth factors and miRNA.

RSD is currently the primary treatment used in cases of hypertension and the results of the present study suggest it may act as a promising candidate for the treatment of heart failure in the future. However, the implementation of RSD in the clinic depends on renal artery catheter ablation, an area where further research is required. Furthermore, the present study focused on myocardial fibrosis and further research is required to validate this method prior to the acceptance of RSD as a clinical treatment of heart failure.

\section{Acknowledgements}

The present study was supported by the Natural Science Foundation of Hubei Province of China (grant no. 2015CFB223). 


\section{References}

1. Brandt MC, Mahfoud F, Reda S, Schirmer SH, Erdmann E, Böhm M and Hoppe UC: Renal sympathetic denervation reduces left ventricular hypertrophy and improves cardiac function in patients with resistant hypertension. J Am Coll Cardiol 59: 901-909, 2012.

2. Ramos-Mondragón R, Galindo CA and Avila G: Role of TGF-beta on cardiac structural and electrical remodeling. Vasc Health Risk Manag 4: 1289-1300, 2008.

3. Liu N and Olson EN: MicroRNA regulatory networks in cardiovascular development. Dev Cell 18: 510-525, 2010.

4. Davies JE, Manisty CH, Petraco R, Barron AJ, Unsworth B Mayet J, Hamady M, Hughes AD, Sever PS, Sobotka PA and Francis DP: First-in-man safety evaluation of renal denervation for chronic systolic heart failure: Primary outcome from REACH-Pilot study. Int J Cardiol 162: 189-192, 2013.

5. Li P, Luo S, Pan C and Cheng X: Modulation of fatty acid metabolism is involved in the alleviation of isoproterenol-induced rat heart failure by fenofibrate. Mol Med Rep 12: 7899-7906, 2015.

6. Hu J, Li Y, Cheng W, Yang Z, Wang F, Lv P, Niu C, Hou Y, Yan Y and $\mathrm{Ge} \mathrm{J}$ : A comparison of the efficacy of surgical renal denervation and pharmacologic therapies in post-myocardial infarction heart failure. PLoS One 9: e96996, 2014.

7. Wu H, Chen L, Xie J, Li R, Li GN, Chen QH, Zhang XL, Kang LN and $\mathrm{Xu}$ B: Periostin expression induced by oxidative stress contributes to myocardial fibrosis in a rat model of high salt-induced hypertension. Mol Med Rep 14: 776-782, 2016.

8. Zhou B, Ma R, Si W, Li S, Xu Y, Tu X and Wang Q: MicroRNA-503 targets FGF2 and VEGFA and inhibits tumor angiogenesis and growth. Cancer Lett 333: 159-169, 2013.

9. Xu C, Yang Q, Xiong H, Wang L, Cai J, Wang F, Li S, Chen J, Wang C, Wang D, et al: Candidate pathway-based genome-wide association studies identify novel associations of genomic variants in the complement system associated with coronary artery disease. Circ Cardiovasc Genet 7: 887-894, 2014.

10. Wang F, Xu CQ, He Q, Cai JP, Li XC, Wang D, Xiong X, Liao YH, Zeng QT, Yang YZ, et al: Genome-wide association identifies a susceptibility locus for coronary artery disease in the Chinese Han population. Nat Genet 43: 345-349, 2011.

11. Eisenhofer G, Friberg P, Rundqvist B, Quyyumi AA, Lambert G, Kaye DM, Kopin IJ, Goldstein DS and Esler MD: Cardiac sympathetic nerve function in congestive heart failure. Circulation 93: 1667-1676, 1996.

12. Verrecchia F and Mauviel A: Transforming growth factor-beta signaling through the smad pathway: Role in extracellular matrix gene expression and regulation. J Invest Dermatol 118: 211-215, 2002.

13. Daniels A, van Bilsen M, Goldschmeding R, van der Vusse GJ and van Nieuwenhoven FA: Connective tissue growth factor and cardiac fibrosis. Acta Physiol (Oxf) 195: 321-338, 2009.

14. Blaney Davidson EN, Vitters EL, Mooren FM, Oliver N, Berg WB and van der Kraan PM: Connective tissue growth factor/CCN2 overexpression in mouse synovial lining results in transient fibrosis and cartilage damage. Arthritis Rheum 54: 1653-1661, 2006.
15. Shi-wen X, Stanton LA, Kennedy L, Pala D, Chen Y, Howat SL, Renzoni EA, Carter DE, Bou-Gharios G, Stratton RJ, et al: CCN2 is necessary for adhesive responses to transforming growth factor-betal in embryonic fibroblasts. J Biol Chem 281: 10715-10726, 2006.

16. Brigstock DR: Regulation of angiogenesis and endothelial cell function by connective tissue growth factor (CTGF) and cysteine-rich 61 (CYR61). Angiogenesis 5: 153-165, 2002.

17. Goren Y, Kushnir M, Zafrir B, Tabak S, Lewis BS and Amir O: Serum levels of microRNAs in patients with heart failure. Eur $\mathrm{J}$ Heart Fail 14: 147-154, 2012.

18. Lijnen P, Petrov V and Fagard R: Transforming growth factor-beta 1-mediated collagen gel contraction by cardiac fibroblasts. J Renin Angiotensin Aldosterone Syst 4: 113-118, 2003.

19. Schultz Jel J, Witt SA, Glascock BJ, Nieman ML, Reiser PJ, Nix SL, Kimball TR and Doetschman T: TGF-betal mediates the hypertrophic cardiomyocyte growth induced by angiotensin II. J Clin Invest 109: 787-796, 2002.

20. Adam O, Lavall D, Theobald K, Hohl M, Grube M, Ameling S, Sussman MA, Rosenkranz S, Kroemer HK, Schäfers HJ, et al: Racl-induced connective tissue growth factor regulates connexin 43 and n-cadherin expression in atrial fibrillation. J Am Coll Cardiol 55: 469-480, 2010.

21. Perlini S, Palladini G, Ferrero I, Tozzi R, Fallarini S, Facoetti A, Nano R, Clari F, Busca G, Fogari R and Ferrari AU: Sympathectomy or doxazosin, but not propranolol, blunt myocardial interstitial fibrosis in pressure-overload hypertrophy. Hypertension 46: 1213-1218, 2005.

22. van Rooij E, Sutherland LB, Thatcher JE, DiMaio JM, Naseem RH, Marshall WS, Hill JA and Olson EN: Dysregulation of microRNAs after myocardial infarction reveals a role of mir-29 in cardiac fibrosis. Proc Natl Acad Sci USA 105: 13027-13032, 2008.

23. Duisters RF, Tijsen AJ, Schroen B, Leenders JJ, Lentink V, van der Made I, Herias V, van Leeuwen RE, Schellings MW, Barenbrug P, et al: Mir-133 and mir-30 regulate connective tissue growth factor: Implications for a role of microRNAs in myocardial matrix remodeling. Cir Res 104: 170-178, 2009.

24. Shlipak MG and Massie BM: The clinical challenge of cardiorenal syndrome. Circulation 110: 1514-1517, 2004.

25. Wang X, Zhao Q, Huang H, Tang Y, Xiao J, Dai Z, Yu S and Huang C: Effect of renal sympathetic denervation on atrial substrate remodeling in ambulatory canines with prolonged atrial pacing. PLoS One 8: e64611, 2013.

26. Clayton SC, Haack KK and Zucker IH: Renal denervation modulates angiotensin receptor expression in the renal cortex of rabbits with chronic heart failure. Am J Physiol Renal Physiol 300: F31-F39, 2011.

27. Hu J, Ji M, Niu C, Aini A, Zhou Q, Zhang L, Jiang T, Yan Y and Hou Y: Effects of renal sympathetic denervation on post-myocardial infarction cardiac remodeling in rats. PLoS One 7: e45986, 2012. 